Como citar este artículo en APA: Pérez Vargas, J.J. \& Cabrera Vásquez, M. E. (2019). Desafíos pedagógicos a la formación en la etapa de noviciado de comunidades religiosas. Cuestiones Teológicas, 46 (106), 272-294 doi: http://doi.org/10.18566/cueteo.v46n106.a04

Fecha de recepción: 10 de mayo de 2019 Fecha de aceptación: 20 de septiembre de 2019

\title{
DESAFÍOS PEDAGÓgICOS A LA FORMACIÓN EN LA ETAPA DE NOVICIADO DE COMUNIDADES RELIGIOSAS ${ }^{1}$
}

\author{
Pedagogical Challenges to Formation during Novitiate \\ Stage within Religious Communities
}

Desafios pedagógicos à formação na etapa de noviciado de comunidades religiosas

\author{
John Jairo Pérez Vargas ${ }^{2}$ \\ María Esmeria Cabrera Vásquez ${ }^{3}$
}

1 Artículo resultado de investigación del proyecto de investigación FODEIN de la Universidad Santo Tomás.

2 Doctorando en Educación de la Universidad Católica de Córdoba, Argentina. Especialista y Magíster en bioética de la Universidad el Bosque, Colombia. Licenciado en Teología de la Pontificia Universidad Javeriana, Colombia. Docente tiempo completo de la Facultad de Educación de la Universidad Santo Tomás. Pertenece al grupo de investigación "Investigación Educativa" de la USTA. Correo de contacto: johnjapeva@gmail.com.

3 Licenciada en Teología de la Universidad Santo Tomás, Colombia. Técnico de Nivel Superior en Enfermería, en el Centro de Formación Técnica ENAC, Chile. Religiosa Sierva de Jesús de la Caridad. Maestra de novicias en Armenia-Colombia. Correo de contacto: esmedej@hotmail.com 


\section{Resumen}

El presente documento de carácter investigativo y reflexivo pretende hacer un análisis a los desafíos pedagógicos de la formación religiosa en la etapa de noviciado. Para ello, se inicia con la especificación de las particularidades de la formación de novicios, de acuerdo con algunos textos magisteriales, ubicando de esta manera el centro de la reflexión. Bajo la descripción de este contexto, los sujetos son objeto de reflexión y de problematización, para lo que se hace un breve análisis que permite al lector reconocer algunas de las particularidades de las personas que ingresan a esta etapa de formación en las comunidades de carácter religioso. A partir de esta enunciación se plantea la reflexión en torno a la pedagogía en el proceso formativo, la cual es analizada con base en los modelos pedagógicos, pero elaborando una crítica al modelo pedagógico tradicional empleado frecuentemente en este tipo de formación. Finalmente se propone una complementariedad desde otros modelos pedagógicos, resaltando al mismo tiempo algunos desafíos que han de estar de manera general presentes en esta importante etapa de formación de la vida religiosa.

Palabras clave: Modelos pedagógicos; Noviciado; Formación; Comunidad religiosa; Pedagogía.

\section{Abstract}

The aim of the article is to analyze the pedagogical challenges of religious formation during novitiate stage. The article begins by presenting the distinctive features of the formation of novices according to some magisterial reference works. Bearing this context in mind, the article presents a brief analysis that makes it possible to recognize some of the distinctive features of the persons that reach this stage of formation within religious communities. Based on this, the article considers the role of pedagogy in religious formation, which is analyzed based on pedagogical approaches. The traditional pedagogical approach for this kind of formation is, therefore, questioned; and a complementarity with some other pedagogical approaches is proposed, highlighting some of the general challenges faced during this relevant stage of religious formation.

Keywords: Pedagogical Approaches; Novitiate; Formation; Religious Community; Pedagogy. 


\section{Resumo}

Esse artigo de pesquisa e de caráter reflexivo tenta analisar os desafios pedagógicos à formação religiosa na etapa de noviciado. Para isso começa-se com a caraterização das particularidades da formação de novicies, segundo alguns textos de referência magistrais, enquanto centro da reflexão. Sob a descrição desse contexto, os sujeitos são objeto de reflexão e de problematização, para isso se faz uma breve analise que permite ao leitor reconhecer algumas das caraterísticas das pessoas que ingressam nessa etapa de formação nas comunidades de caráter religioso, a partir dessa enunciação se propóe refletir sobre a pedagogia no processo formativo. Essa é analisada com base nos modelos pedagógicos, depois de elaborar uma critica ao modelo pedagógico tradicional, que foi usado muitas vezes nesse tipo de formação. Isso no intuito de propor um complemento a essa pedagogia com outros modelos pedagógicos para ressaltar, assim, alguns desafios que têm que estar presentes de modo geral nessa importante etapa de formação da vida religiosa.

Palavras chave: modelos pedagógicos; noviciado; formação; comunidade religiosa; pedagogia.

\section{Introducción}

La formación del clero y de personas consagradas es una cuestión que ha inquietado desde hace mucho tiempo a la Iglesia, y en particular a las órdenes y comunidades religiosas, ya que la educación es el motor que posibilita, al interior de la Iglesia católica, que la estructura y los ideales de las comunidades, a la luz de la Iglesia universal, se mantengan con base en sus principios fundacionales, identitarios $\mathrm{y}$ de fe, y perduren en el tiempo.

De manera general, se puede asumir que la comprensión interna de la Iglesia en su aspecto formativo es básicamente de carácter relevativo, es decir que al ser una institución que perdura en el tiempo, la transmisión del conocimiento propio que caracteriza los intereses identitarios de carácter eclesial, dogmas y oficios propios de la vida religiosa, son ejes fundamentales 
de la formación. A la vez, y en esta dinámica formativa, es necesario que sus miembros se faculten para que, con el paso del tiempo, puedan asumir funciones que otros han venido desarrollando, logrando así la supervivencia en el trasegar del tiempo.

Se reconoce que el lugar de la formación en el interior de la vida religiosa es esencial al enmarcarse en una institucionalidad y en una misión identitaria propia del cristianismo. En efecto, la formación en la vida religiosa propende por esta labor, a la vez que plantea desafíos particulares en la transición de épocas y lugares en los cuales la Iglesia asume su misión.

Con este panorama alrededor de la formación al interior de la Iglesia, el presente artículo propone focalizar su atención en la formación de novicios, a partir de un trabajo investigativo de corte documental, que propone dar cuenta de un diagnóstico y algunas recurrencias en el fenómeno educativo en la formación impartida en la etapa del noviciado, identificando el papel de la pedagogía en esta etapa y de la vida religiosa en general.

Para ello, la ruta que se plantea en el presente texto está comprendida por un punto de partida ubicado en la aclaración del contexto específico en el cual se desarrolla la investigación y la metodología empleada; posterior a ello se procederá a la identificación de las características e ideales de la formación en la etapa de noviciado. Con ello se analiza el impacto de algunos modelos pedagógicos en esta etapa formativa, preparando así el camino para formular algunos desafíos que la pedagogía puede plantear a la formación de novicios.

\section{Contexto y metodología de la investigación}

La presente investigación se ubica en el campo de la educación al interior de la Iglesia, limitando su horizonte de trabajo a la formación en la vida religiosa, en la etapa de noviciado. La cual, de acuerdo al Código de Derecho Canónico, tiene como finalidad que "los novicios conozcan mejor la vocación divina, particularmente la propia del instituto, que prueben el modo de vida de éste, que conformen la mente y el corazón con su espíritu, y que puedan ser comprobadas su intención y su idoneidad" (n. 646). 
En este sentido, el noviciado se caracteriza por ser una de las primeras etapas de formación dentro de la vida religiosa, en la cual los aspirantes a la comunidad o congregación entran en un proceso de discernimiento y conocimiento de la implicación que tiene asumir un estilo de vida consagrado, en el contexto particular de la misión o carisma propio de la comunidad religiosa. Con base en esto, se presume y asume que todo interesado en hacer parte de la vida religiosa deberá pasar por esta etapa.

La importancia de la etapa del noviciado, en este sentido, es esencial para la asimilación, reflexión e interiorización de la vocación, y del compromiso que el novicio o novicia desean adquirir de manera voluntaria ante la comunidad religiosa y la Iglesia. En esta medida, la formación que se imparte allí es esencial para la comprensión de estas implicaciones. Por esto, el presente artículo busca investigar y reflexionar a propósito de esta etapa de formación, evidenciando los posibles aportes y retos que trae la pedagogía al mediar en esta etapa.

En este punto es pertinente aclarar por qué se habla reiterativamente de formación y no de educación, o exclusivamente de pedagogía. Esto se da porque al interior de la estructura eclesial y magisterial suele utilizarse de manera mucho más recurrente la referencia a "formación" que a la noción de "educación". De ello dan cuenta documentos magisteriales que se enfocan de manera precisa a esta temática, como el Código de Derecho canónico, el Concilio Vaticano II, la exhortación apostólica Vita Consecrata, la declaración Perfectae Caritatis, entre otros.

Además de lo enunciado, la palabra formación en su raíz latina está compuesta por la palabra forma que significa imagen, figura, configuración, constitución y el sufijo -ción, el cual significa acción y efecto. Con ello se asume que la raíz sugiere el hecho de dar forma. En este sentido, y bajo una visión teológica, entendiendo que los cristianos estamos llamados a ser Alter Christus, los ejercicios formativos han de suponer un dar forma de Jesucristo, no en lo que respecta a su naturaleza divina, sino en las posibilidades de ser a partir de lo propio y limitado de la naturaleza humana. Con este presupuesto se comprende que el uso de la palabra formación no es azaroso, sino que en el fondo hay impresa una clara intencionalidad de carácter teológico. 
Con todo lo anterior, es importante aclarar que aunque el presente artículo centra su intencionalidad analítica y reflexiva en el noviciado, habrá referencias propias a la formación en general, dado que el tema de la formación en los discursos magisteriales suele abordarse en la mayoría de las veces de manera genérica, impactando todos los campos de formación, bien sean a nivel social o en lo propio que a la vida religiosa compete.

Con esta aclaración, se puede inferir que aunque el centro de la reflexión sea focalizado la mencionada etapa, las reflexiones o aportes que se hagan en este campo tampoco son exclusivos de la formación en los noviciados. En este sentido, los diagnósticos, retos, desafíos, entre otros, podrán ser insumo de reflexión y aporte a distintas etapas formativas presentes en distintos momentos al interior de la vida religiosa.

A nivel metodológico, el presente texto se ubica en el paradigma cualitativo, en tanto que aborda un tema de investigación que parte del análisis de un problema de carácter social y humano (Creswell, 1998). Para la presente investigación está claro que el paradigma se aborda desde la educación, la cual se analiza desde los desafíos de carácter pedagógico en la formación.

La perspectiva epistemológica, por su parte, se constituye como aquellos lentes con los cuales se aborda el tema de investigación, otorgando una ruta de análisis que permite la comprensión del hecho, fenómeno o categoría a analizar (Pérez, Nieto y Rodríguez, 2019). Para el caso de la presente artículo se hace un abordaje de corte hermenéutico, en cuanto que se busca efectuar un análisis profundo en la materia, indagando el sentido y fundamento de los ítems teóricos (Marín, 2016, p. 131).

La hermenéutica, en este contexto, ofrece la posibilidad de analizar la formación que se vive en la etapa de noviciado por medio de la interpretación alrededor de los elementos que circundan estos procesos. En este sentido, la experiencia del intérprete, las precomprensiones, los contextos y la aproximación a la realidad a analizar han de determinar las apreciaciones y reflexiones que puedan emerger en el desarrollo del documento. 
El tipo de investigación es de carácter documental, la cual se caracteriza por la búsqueda, organización, lectura, clasificación, comparación y presentación de ideas, temas o categorías encontradas en los documentos (Páramo, 2013). En lo propio de la investigación, la búsqueda de información se fundamentó en primer lugar en la consulta a partir de los documentos magisteriales, los cuales hacían referencia a la formación o al noviciado y educación en distintos niveles. De igual manera se complementó esta base documental a partir de indagaciones realizadas en bases de datos especializadas que abordaran elementos afines a la temática a analizar y permitieran completar las fuentes y datos.

Una vez recopilados los documentos se procedió a la organización y sistematización de los mismos. Para ello se emplearon las Rejillas de Análisis Documental (RAD), las cuales permitieron organizar los textos por temáticas, ideas, argumentos y categorías, otorgando de esta manera insumos para la sistematización de la información y presentación de la misma en el cuerpo final del artículo.

Bajo esta ruta metodológica y contextual, el presente texto inicia haciendo un diagnóstico y presentación alrededor del tema de la formación propia de los noviciados, detallando algunas intencionalidades e intereses que se pueden detectar a la luz de algunos documentos magisteriales. Así, se abre la oportunidad para poder abordar la temática desde el escenario propio de las particularidades de la persona que ingresa a los noviciados y la lectura de algunos modelos pedagógicos que son más preponderantes en la formación novicial. Al finalizar, se podrán proponer unos desafíos que en el contexto pedagógico se plantea a la formación de este estilo de vida.

\section{Formación en los noviciados}

El noviciado, de acuerdo con el Código de Derecho Canónico en el canon 646, es considerado como una etapa fundamental en la aspiración a la vida consagrada, pues es allí donde los aspirantes se aproximan a la comunidad religiosa, conociendo de la misma, y en actitud de discernimiento se adentran 
a la experiencia de búsqueda y corroboración de su vocación, con la vivencia propia de la vida en comunidad, bajo el carisma y particularidades del respectivo instituto. Así se expresa en Congregación para los institutos de vida consagrada y las sociedades de vida apostólica (1990):

En otros términos, teniendo en cuenta la diversidad de carismas e institutos, se podría definir el fin del noviciado como un tiempo de iniciación integral al género de vida que el Hijo de Dios asumió y que Él nos propone en el Evangelio, en uno u otro aspecto de su servicio o de sus misterios (n. 45).

En este sentido, la etapa de noviciado es un tiempo esencial en la vida religiosa, en la cual los novicios van a conocer un poco sobre este estilo de vida, fundamentado en un encuentro consigo mismo, con sus hermanos y con Jesucristo. Dicho encuentro lleva a la persona a adentrarse en ella misma para iniciar un camino de reflexión, interiorización y de transformación existencial, el cual le permite ahondar en el conocimiento de su persona y su misión, para luego salir al encuentro del otro y lo otro, y al reconocimiento del prójimo en un mundo diverso.

Ahora bien, este proceso se ve atravesado por una etapa o intencionalidad formativa, la cual se enmarca en el discernimiento, el descubrimiento y comprobación de la vocación y la preparación para la vivencia gradual de la perfección propia del instituto (CIC, 652). En palabras de san Juan Pablo II: "La formación, por tanto, debe abarcar la persona entera, de tal modo que toda actitud y todo comportamiento manifiesten la plena y gozosa pertenencia a Dios, tanto en los momentos importantes como en las circunstancias ordinarias de la vida cotidiana" (VC 65).

En este sentido, dicha formación apunta a un proceso de cambios que abarca a toda la persona y que dura en el tiempo, causando un impacto personal, congregacional, eclesial y social, procurando un camino de concienciación y de liberación (Pérez, González, y Rodríguez, 2017). Por consiguiente, se sostiene que la formación en el noviciado debe ser sólida, como un edificio bien construido, para soportar los cambios de la naturaleza y las inclemencias del tiempo. Es así que no se concibe una formación a medias, en donde pareciera que se mide el tiempo y los resultados y no los procesos, pues estos últimos son esenciales en todo acto formativo. 
Por tanto, apostar por una formación que ayude adquirir cada vez más esa capacidad emprendedora e inteligente, reflexiva, ágil e ingeniosa que hace crecer con la vida y gracias a la vida, y que coadyuva a librarse de ciertas rigideces personales que impiden cualquier crecimiento, ante Dios y ante los hombres, es un objetivo esencial en todo acto formativo (Amadeo, 2003, p, 4).

El Concilio Vaticano II (1965) en el "Decreto Perfectae Caritatis" reconoce que: "La renovación de los institutos religiosos depende principalmente de la formación de sus miembros (n. 18). Dicha formación implica un proceso de toda la vida que tiene luces y sombras. Por lo cual un especial énfasis en la etapa de noviciado ayuda a la consolidación de elementos que contribuyen de especial manera a formar para la vida religiosa y los desafíos que tanto la institución como las personas han de enfrentar.

De acuerdo al Código de Derecho Canónico, "Para atender a la formación de los novicios deben destinarse miembros cuidadosamente preparados, que, sin estar impedidos por otros trabajos puedan cumplir sus funciones con fruto y de manera estable" (n. 651). Según Juan Pablo II, se comprende que Dios Padre, en el don continuo de Cristo y del Espíritu, es el formador por excelencia de quien se consagra a Él. Pero en esta obra Él se sirve de la mediación humana, poniendo al lado de los que Él llama a personas y maestros capaces de enseñar y guiar de manera asertiva a los novicios y que contribuyan en el pleno desarrollo de su personalidad (CV 66).

Con lo anterior, una importante consideración que se debe tener a nivel formativo es la del maestro, quien ha de ser una persona idónea, en cuanto que cumple con ciertas características. Esta idoneidad no es solo a nivel cognitivo o espiritual, sino que ha de estar comprendida por la articulación de ambas, entendiendo de esta manera que el maestro juega un papel esencial en toda etapa formativa.

En conformidad con el Código de Derecho Canónico en su canon 650 demanda que "la finalidad del noviciado exige que los novicios se formen bajo la dirección de un maestro, según el plan de formación que debe determinar el derecho propio". De tal modo que este maestro "ha de ser un miembro del instituto profeso de votos perpetuos y legítimamente 
designado" (CIC, 651). A su vez, el Código establece que de ser necesario, a este maestro se le podrán asignar también ayudantes que bajo la dirección de él, colaborarán en los ejercicios formativos, la única condición es que estos deben estar cuidadosamente preparados, no contar con algún impedimento y puedan cumplir a cabalidad con las funciones asignadas (CIC, 651).

Es por ello que no cualquier persona es la idónea para acompañar estos procesos formativos, pues además de los requerimientos canónicos, a nivel pedagógico se necesitan varias características que le otorguen la posibilidad de impacto y de transformación social (Giroux, 1990, p. 171). Poseer algún conocimiento en la materia es fundamental en cuanto que con base en este se "[...] puede afectar el cómo los profesores critican los libros [...] a cómo seleccionan el material para enseñar, a cómo estructuran sus cursos y a cómo conducen la instrucción" (Grossman, Wilson y Shulman, 2005, p. 13), configurando de esta manera el acto y proceso de la enseñanza.

El maestro que acompaña los procesos formativos o sus colaboradores, especialmente en esta etapa, han de ser personas preparadas, que gocen de experiencia, una sólida vida espiritual, un fuerte conocimiento en aquello que desea transmitir, inducir o producir en sus estudiantes. En esta etapa los ejercicios de concienciación han de privilegiarse en tanto que con esta formación se juega mucho de las expectativas y comprensiones que se hagan sobre la vida religiosa. En este sentido es importante tener presente que "tanto el docente como el estudiante se presentan como agentes orgánicos del acto educativo, quienes a través de su interacción histórica y cultural, posibilitan la construcción de saberes" (Santamaría, Quitián y Orozco, 2016, p. 2017).

Es importante tener en cuenta que en el noviciado la formación se caracteriza por el abordaje de distintas áreas, tales como: la espiritual, apostólica, comunitaria, intelectual, ecológica, entre otras; con el objetivo de formar personas consagradas que sirvan al pueblo de Dios confiado a ellas, tal como lo hizo Jesucristo en su vida pública (Hechos 10, 38). Al respecto, san Juan Pablo II afirma que: "Se ha de prever, por tanto, una formación humana, cultural, espiritual y pastoral poniendo sumo cuidado en facilitar la integración armónica de los diferentes aspectos (VC 65). Todas estas áreas deben ir articuladas, considerando a la persona como un todo en proceso de construcción y en constante cambio. 
Este panorama interpela aún más el perfil del maestro, en tanto que no cualquiera es apto para formar en estas dimensiones, temáticas o aspectos esenciales para la vida consagrada. Con ello, la improvisación no es una opción, y la falta de experiencia o experticia puede generar vacíos, incertidumbres o carencias en la manera en que se comprende y asume la vida religiosa en general. Sobre todo porque, como recuerda Mahecha, Cubillos y Pico (2018), existe una necesidad para cada ser humano en proceso de desarrollo y de educación, que se concreta en involucrar de manera intencionada dentro de sus búsquedas aquella que se relaciona con la formación de la interioridad y la espiritualidad, lo que implica no limitarse a aspectos contemporáneos o de recuerdos no aceptados, sino contemplar el conjunto de la historia vital previendo que la intervención de acompañamiento favorezca la conexión, entre la persona multidimensional y su vocación y proyección a futuro.

Sumado a ello, con este recorrido se puede identificar una clara responsabilidad ética en el ejercicio formativo, pues la definición de educación o formación reviste por sí misma de una carga ética y moral asociada su labor intrínseca (Hernández, 2010, p. 216). Por lo que la institución educadora o formadora ha de cargar un lugar privilegiado con esta responsabilidad la cual se ha de articular junto con la figura y papel del maestro y del alumno.

El ejercicio formativo en la etapa de noviciado ha de estar condicionado a la responsabilidad que recae sobre la institución, el maestro y el estudiante. La institución, que debe presentar de manera precisa y clara los ideales formativos, proponiendo recursos, búsquedas formativas amparadas en intenciones curriculares que permitan vislumbrar una clara intencionalidad formativa. Por su parte, el maestro debe ser una persona preparada, con conocimiento en la materia y con capacidad articulada de transformación, transmisión y reflexión. Finalmente, el estudiante quien debe disponer de sí mismo en condición de apertura, dedicación y voluntad para que el acto educativo conduzca a los fines deseados.

Finalmente, con lo expresado en este apartado, se pueden inferir algunas preguntas que han de seguir motivando la reflexión y la investigación en esta materia como, por ejemplo, ¿cuál es la formación que se imparte en los noviciados en el siglo XXI? ¿Cuáles son los propósitos y las metas de 
formación en el noviciado? ¿Qué tipo de novicios se quiere y necesita formar? ¿Para qué instituto o congregación se está formando a los futuros profesos o religiosos? ¿Cómo se puede formar a un novicio o novicia en el siglo XXI? ¿Quién debe liderar el proceso formativo en el noviciado? ¿Con qué técnicas, procesos, métodos, herramientas cuentan las instituciones o congregaciones para formar a las novicias? ¿Cómo se debe evaluar el proceso formativo durante la experiencia del noviciado? ¿Qué tipo de consagrados se quiere formar hoy? A lo que es pertinente enunciar que la vida consagrada debe adaptarse a cada época y a cada contexto para que dé frutos abundantes (Mateo 7, 15-20), y responda a las necesidades concretas que han de enmarcar la misión de la Iglesia en los contextos.

Cada congregación debe abrirse a nuevos horizontes de formación y por ende estar en actitud de acogida a los signos de los tiempos, tal como lo propone el Concilio Vaticano II, para discernir y comprender las necesidades más profundas del momento y responder de modo asertivo a las expectativas. Corresponde a cada congregación dar las directrices apropiadas para que también en el siglo XXI la vida consagrada prodigue al mundo su mensaje mediante consagrados que sean servidores y alegres, adaptados y en respuesta a las necesidades y demandas de su época y lugar de acción.

\section{Algunas consideraciones de quienes ingresan a los noviciados}

El presente apartado pretende evidenciar los perfiles y características de las personas que ingresan a los noviciados, no con ánimo de universalizar o agotar el perfil del aspirante, sino como la oportunidad de ofrecer un panorama de comprensión que de manera general ilumine o interpele los procesos formativos que están presentes en la etapa de noviciado.

Las personas que integran la etapa de noviciado, dependiendo de la comunidad o instituto religioso, en algunas ocasiones hacen previamente un curso o ejercicio de prenoviciado, el cual alerta de la vida comunitaria y funge como un filtro en el cual las personas inquietas en su vocación pueden hacerse una idea de las implicaciones de la vida religiosa. Posterior a esta etapa, viene de manera formal la del noviciado. 
En los cánones 641-645, el Código de Derecho Canónico establece algunas condiciones que deben tenerse en cuenta sobre las admisiones al noviciado. Así, se establece que la admisión o no a la etapa de noviciado es determinada por los Superiores mayores (CIC, 641). Se admitirán quienes "[...] además de la edad necesaria, tengan salud, carácter adecuado y cualidades suficientes de madurez para abrazar la vida propia del instituto; estas cualidades de salud, carácter y madurez han de comprobarse, si es necesario, con la colaboración de peritos" (CIC, 642).

$\mathrm{Al}$ interior de las invalidaciones que restringen el ingreso al noviciado se contemplan los siguientes:

1 quien aún no haya cumplido diecisiete años; 2 un cónyuge, durante el matrimonio; 3 quien se halla en ese momento ligado por un vínculo sagrado con algún instituto de vida consagrada o está incorporado a una sociedad de vida apostólica, sin perjuicio de lo que prescribe el c. 684; 4 quien entra en el instituto inducido por violencia, miedo grave o dolo, o aquel a quien el Superior admite inducido de ese mismo modo; 5 quien haya ocultado su incorporación a un instituto de vida consagrada o a una sociedad de vida apostólica (CIC, 643).

Sumado a lo anterior, cada instituto o comunidad religiosa podrá establecer o imponer algunas otras condiciones bajo el derecho propio.

En cuestión de tiempos, el noviciado dura un año, y debe ser efectuado en las instalaciones de la misma comunidad (CIC, 648). De igual manera, el novicio podrá abandonar el proceso de manera libre cuando lo estime necesario, o en dado caso la autoridad competente podrá despedirle. 1 finalizar la etapa, el novicio habrá de ser admitido a la profesión temporal en caso de idoneidad, si no es así podrá ser despedido o se podrá efectuar una prórroga en el proceso de noviciado (CIC, 653).

De manera particular, es importante reconocer que los novicios vienen de un contexto singular y que para cumplir con esta etapa formativa, suelen agruparse en casas de formación ubicadas en ciudades medianas y grandes, de tal modo que para muchos supone alejarse de sus contextos propios de origen y adaptarse a otras dinámicas de realidad. Esto trae consigo un proceso de separación, de adaptación y de nuevos aprendizajes. 
Es igualmente importante reconocer que la diversidad de personas y perfiles que aspiran a hacer parte de esta etapa de formación es amplia, y trae sus contingencias, en cuanto que en varios de los casos hay muchas dificultades que subyacen a la persona, tales como ambientes familiares disfuncionales, ausencia de los progenitores en el hogar, violencia intrafamiliar, abuso y violación sexual. Asimismo, las adicciones, tales como la droga, el alcohol, desórdenes alimenticios, entre otros tantos factores que plantean serios desafíos a la formación de la persona (Castillo y Gamboa, 2012).

Ante esta posible diversidad de perfiles, los procesos formativos no pueden ser ajenos, en cuanto que las realidades humanas son interpeladoras de la educación. En este sentido, la tarea formativa no ha de cumplir una función exclusivamente transmisionista, sino que ha de ser la promotora de procesos de reivindicación de la persona que le permitan estados de liberación de distintas condiciones que le oprimen (Vargas, Arcila y Robayo, 2017).

En esta descripción se debe tener presente que con lo mencionado anteriormente no se pretende desconocer que hay realidades particulares que posibilitan la comprensión de los perfiles de ingreso a la etapa de formación de noviciado, es por ello que las instituciones o personas encargadas de estos procesos formativos deben tener en consideración distintos aspectos particulares de los futuros novicios tales como aspectos cognitivos, praxiológicos, axiológicos, pastorales, espirituales entre otros, los cuales sin lugar a dudas deberán ser considerados en los procesos formativos que se asuman al interior del noviciado.

\section{Los modelos pedagógicos y la formación de novicias}

Uno de los ítems más importantes en todo proceso educativo está relacionado con los modelos pedagógicos que subyacen en la dinámica enseñanzaaprendizaje, pues estos han de marcar el dinamismo, apropiación y el ritmo sobre el que ha de fluir el conocimiento y las intencionalidades formativas que rondan los espacios educativos. Es por ello que este apartado se plantea la problematización alrededor de este importante tema pedagógico, propiciando un espacio de reflexión e interpelación que desemboque en el planteamiento de desafíos pedagógicos, los cuales han de estar atravesados 
por las consideraciones anteriormente expuestos en relación a la formación en la etapa de noviciado.

Antes de iniciar este abordaje conviene precisar que "todo modelo pedagógico tiene su fundamento en los modelos psicológicos del proceso de aprendizaje, en los modelos sociológicos, comunicativos, ecológicos o gnoseológicos" (Ocaña, 2013, p. 40), de ahí que la relación entre estas áreas del saber y la pedagogía sea fundamental para comprender el alcance y el impacto de los modelos pedagógicos.

Sumado al aporte de diversas ciencias, los modelos pedagógicos son considerados como representaciones teóricas y prácticas que ayudan de una $\mathrm{u}$ otra manera a iluminar la realidad y transformarla, puesto que parte de una comprensión del hombre y cumple un ideal o meta trazada, creando estrategias e instrumentos apropiados para conseguir dicha meta. A partir de esta noción, ningún proceso educativo se puede comprender de manera aislada a algún modelo pedagógico.

Se reconoce la necesidad de los modelos pedagógicos en el acto formativo de las futuras personas consagradas, en razón de las intencionalidades formativas alrededor de la vocación, procesos comunitarios, conocimiento eclesial, religioso, y especialmente del cultivo de la espiritualidad entendida como "la praxis natural del cultivo de la interioridad humana, [...] búsqueda del conocimiento de sí mismo y el cuidado del desarrollo personal, fundamentados en las construcciones de sentido de vida" (Naranjo y Moncada, 2019, p. 116). Los cuales han de propiciar en los novicios y novicias una formación reconocida como integral y significativa que contribuya al discernimiento y conocimiento de la vida que se desea asumir.

$\mathrm{Al}$ interior de los procesos formativos y el reconocimiento de la importancia de los modelos pedagógicos en el acto educativo, emergen parámetros que han de ser tenidos en cuenta a la hora de implementar un determinado modelo. De esta manera, en primera instancia se trae a colación el papel que ha de desempeñar el formando en su educación. Un fragmento de la Conferencia Española de Religiosos respecto a la responsabilidad en la formación, insiste en que: 
La primera responsabilidad en su crecimiento vocacional la tiene la misma persona en formación: 'el llamado está pues continuamente invitado a dar una respuesta atenta, nueva y responsable' [...]. Si el sujeto no está motivado para recorrer un largo camino y para aceptar algún cambio en su persona, todos los esfuerzos que se hagan desde fuera serán muy poco duraderos (2015, p. 464).

De lo anterior se puede inferir que el primer responsable en los procesos de formación ha de ser la persona misma, por ello la educación o pedagogía empleada ha de trabajar en el reconocimiento de la persona como agente de sus procesos formativos, generando así conciencia de autonomía y responsabilidad. Sumado a lo anterior, y en un segundo momento, el modelo pedagógico empleado tendrá el desafío de impactar los sujetos que consecuentemente generarán aprendizajes e interpelaciones, los cuales son esenciales para el desarrollo de la persona, el reconocimiento de su vocación y la seguridad del camino que se desea emprender.

Para ello, los conocimientos, las emociones, el fortalecimiento de la vida espiritual y comunitaria, entre otros, han de ser el centro de la formación y las mediaciones pedagógicas. De tal modo que no basta con tener planes de formación aparentemente bien diseñados y normas aparentemente bien establecidas, sino que aquí se puede problematizar alrededor de preguntas tales como las que se enuncian a continuación: ¿cómo enseñan los maestros en los noviciados? ¿Qué estrategias utilizan? ¿Se tiene en cuenta los contextos de los novicios/as? ¿Cómo aprenden los novicios/as? ¿Cómo contribuye el maestro, la comunidad y la sociedad a los procesos formativos? ¿Qué estrategias han de apoyar y contribuir a esta etapa particular de formación? Estas y muchas más preguntas se pueden formular alrededor de la pedagogía y de la formación en la etapa de noviciado.

Así, se propone reconocer el importante lugar de los modelos pedagógicos en el abordaje de estas problematizaciones, para ello es esencial reconocer que los mismos ayudan a iluminar la realidad y a comprenderla, dando respuesta a las necesidades de cada momento, al tipo de hombre o mujer que la sociedad y la Iglesia demandan. En la actualidad podemos hablar de varios modelos, a saber, algunos de ellos son: tradicional, activo, conductista, constructivista, social, entre otros tantos, (Flórez, 2005). De 
una u otra manera, alguno o varios de estos modelos están presentes en los procesos formativos de la educación al interior de la vida religiosa y juegan un papel fundamental en procesos formativos de los novicios.

Entre estos modelos, en la historia de la formación de la Iglesia al interior de la vida religiosa hay un modelo pedagógico que resalta con gran preponderancia y es el tradicional. El cual se caracteriza por ser un modelo de carácter transmisionista, en donde las alumnos son meros receptores de información, no hay participación en el grupo y la relación del maestro con el estudiante es vertical, él es el que sabe y, por ende este se preocupa más por enseñar contenidos e ideas que por otros procesos que circundan la formación en las personas. Como bien lo plantea Zubiría (2010): "el maestro monopoliza la palabra y la acción, centraliza el poder, la autoridad y las decisiones; él dice qué, cuándo y cómo hacerlo" (p. 139).

No es azaroso el lugar de este tipo de modelo en la tradición educativa eclesial, puesto que el mismo fue la configuración de un amplio proceso de articulación histórica, la cual "es seguidora de la enseñanza directa y severa, predeterminada por un currículo inflexible y centrado en el profesor" (Rodríguez, 2013, p. 44). Por ello, el modelo tradicional es resultado de la configuración jerárquica de la Iglesia, la cual requería de la transmisión de conocimientos y dogmas que permitieran la prevalencia en el tiempo.

Con base en lo anterior, cabe preguntarse si en un noviciado aún hoy tiene cabida un modelo que en muchas de las ocasiones no ayuda a crecer en libertad de expresión, a tener juicios críticos sobre el conocimiento y también sobre quienes los imparten. Si precisamente lo que se desea es que la formación sea un espacio en donde la persona se desarrolle en todas sus dimensiones, no solo debe ser receptora pasiva de información, sino que se necesita que aprenda a interactuar, convivir, dialogar, hablar, pensar, discrepar, hacer un juicio, entre otras cosas propias de una persona consagrada del siglo XXI.

La formación amparada en modelos pedagógicos, como el tradicional, está llamada a repensarse o articularse con otros modelos, de tal modo que se evidencie un aporte sustantivo al ejercicio del maestro y la dinámica enseñanza-aprendizaje. En esta línea, modelos como el social o cooperativo 
están llamados a entrar en escena, pues este modelo se caracteriza por privilegiar la participación en grupo, donde se puede dialogar con el maestro y compañeros, discutir temas, y la formación se centra en el desarrollo de la persona. En palabras de Flórez (2005): “[...] el trabajo en grupo estimula, ayuda a los estudiantes a refinar su trabajo y darse coraje y apoyo mutuo para comprometerse en la solución de los problemas comunitarios" (p. 196).

Esto contribuye de manera significativa en la formación de la etapa de noviciado, puesto que favorece la comunicación, la valoración de lo diverso, los aprendizajes desde otras perspectivas y contextos, entre otros. De esta manera se crece en habilidades, capacidades y competencias que posibilitan el entrar en contacto con los demás y a resignificar al otro como posibilidad de aprendizaje y de trascendencia.

Es importante reconocer que una de las realidades complejas del ser humano es aprender a trabajar en grupo e interactuar sin privilegiar intereses personales, lo cual indica que es un camino por alcanzar y por ende se transa como un desafío para el maestro y estudiantes. De esta manera, se enfatiza la necesidad de contar con este modelo planteado en la formación de los futuros consagrados, llamados a interactuar con el prójimo, a tener una comunicación transparente, por lo cual se insta a capacitar para la comunicación y el diálogo asertivo con el mundo actual.

Asimismo, se puede decir algo del constructivismo, como un modelo que aporta en un nivel significativo a los procesos e intencionalidades formativas en un noviciado, en la medida que se tiene en cuenta los saberes de los formandos, y desde allí se construye el conocimiento. En palabras de Zubiría (2010): "[...] las ideas se relacionan sustancialmente con lo que el alumno ya sabe. Los nuevos conocimientos se vinculan, así; de manera estrecha y estable con los anteriores" (p, 166). Esto permite crear un conocimiento responsable, habilitando a la persona a apropiarse del mismo y a construir de manera autónoma nuevos saberes sin que resulten ser impuestos. Bajo este modelo, los aprendizajes a corto plazo de los novicios deben llevar a un replanteamiento de las estrategias utilizadas, puesto que sin procesos o métodos asertivos la formación no devendrá en su quehacer apostólico y por ende en su crecimiento integral, no aportando significativamente a su misión, a la institución y por ende a la Iglesia. 
En este orden de ideas, Karl Rahner (ctd. por Berríos, 2012) afirma sobre el método antropológico, la necesidad que hay de contar con los saberes previos del sujeto creyente para cada uno de sus procesos. Esto interpela de manera seria la formación y la importancia de ello en el quehacer pedagógico, de tal modo que el centro se ha de ver desplazado de factores teóricos que sin duda resultan importantes, a centrarse en la realidad humana, vinculando así un nuevo horizonte de comprensión. Generalmente no se tiene en cuenta esta realidad o a veces sin querer hacerlo de manera voluntaria se corre el riesgo de pasar por alto esta faceta tan importante, provocando que los resultados pedagógicos arrojen unas consecuencias que puede en muchos de los casos y sentidos no impactar la vida y la persona humana.

\section{Conclusiones}

Con los elementos planteados anteriormente, se pueden establecer algunos retos y desafíos pedagógicos a la formación en la etapa de noviciado de comunidades religiosas. De esta manera se evidencia que todo acto educativo necesita de una pedagogía que ayude a comprender la realidad del ser humano, su proceso inacabado, a la vez que ha de aportar en el aprendizaje y reconocimiento de lo diverso, aceptando y respetando la diferencia.

Esta pedagogía ha de ayudar a los novicios en su formación a ser mejores personas, a sentirse personas activas de la sociedad, a contribuir en su transformación y no ser meros espectadores de la realidad. En las intenciones formativas del noviciado no basta con ser buenas personas; se necesitan seres consagrados con valores y principios que luchen por la justicia y el bien de todos, en donde nadie se sienta explotado y excluido; a la vez que estas personas han de ser conocedoras de la realidad de su pueblo y de las bases cristianas que propenden por lecturas evangélicas de las distintas contingencias humanas.

Todo esto se puede alcanzar si se hace una opción por un modelo pedagógico apropiado para tal etapa e intencionalidades formativas, en palabras de Zubiría (2010): "Un modelo que concluya que la finalidad de la educación no puede estar centrada en el aprendizaje, como desde hace siglos 
ha creído la escuela, sino en el desarrollo" (p. 196). Dicho desarrollo ha de entenderse como el modo de sentirse bien con los demás, de interactuar, de amar y sentirse amado, de sentirse libre, esto implica un trabajo de manera integral y continua, puesto que es un proceso inacabado y constante.

Respecto de los maestros, Julián de Zubiría (2010) hace alusión a estas responsabilidades afirmando que:

Como educadores, somos responsables del desarrollo de la dimensión cognitiva de nuestros estudiantes; pero tenemos iguales responsabilidades en la formación de un individuo ético que se indigne ante los atropellos, se sensibilice socialmente y se sienta responsable de su proyecto de vida individual y social (p. 196).

Sumado a ello, san Juan Pablo II plantea unos aportes interesantes al respecto, en donde no solamente se debe contar con elementos espirituales sino también con herramientas humanas; para ello, él afirma lo siguiente: "A las luces de la sabiduría espiritual añadirán también aquellas que provienen de los instrumentos humanos que pueden servir de ayuda, tanto en el discernimiento vocacional, como en la formación del hombre nuevo auténticamente libre" (VC 66). Por lo tanto, si el noviciado entra en diálogo con la pedagogía facilita el proceso de los novicios y las novicias, contribuyendo a que se forme desde el noviciado un sujeto capaz de comunicarse con su entorno de manera libre y responsable.

Otro elemento o reto de la pedagogía en el contexto de los noviciados en la vida religiosa es el de vigilar, apoyar y garantizar la formación que se brinda a los novicios, como también investigar el quehacer de los maestros, con el fin de ir perfeccionando los modelos pedagógicos que respondan a las necesidades particulares de los sujetos en formación. Los cambios en toda sociedad e institución son constantes, ante ello se requiere también discernimiento y conocimiento de las nuevas transformaciones, culturas, sus realidades y necesidades personales y comunitarias, sociales, y religiosas.

Se puede comparar la formación de los novicios como un campo de construcción en donde se precisa de varios elementos, tales como herramientas adecuadas, materiales óptimos, personal capacitado, obreros idóneos, arquitectos e ingenieros competentes, entre otros. Asimismo, la 
pedagogía ha de custodiar la formación en los noviciados, como también de los maestros y su entorno. Evidentemente, esto lleva a crear estrategias de crecimiento mutuo, generando cambios y transformaciones que impacten el ámbito social.

En la actualidad, como bien menciona Rafael Flórez (2005), no se puede enseñar sin pedagogía. A la hora de la verdad y en caso de necesidad, cualquiera enseña; pero enseñar de manera adecuada es un arte más difícil que exige tener claro para dónde se va, cómo es que la persona aprende y se desarrolla, qué tipo de experiencias son más pertinentes y eficaces para la formación y el aprendizaje, y con qué técnicas y procedimientos es más efectivo enseñar ciertas cosas. Dicho de otro modo, un modelo pedagógico es un constructo entre experiencias, relaciones y oportunidades vinculares, que ilumina no solo a las instituciones sino también al quehacer educativo del maestro, en donde le permite plantearse y abordar preguntas propias de la pedagogía tales como "¿A quién voy a enseñar? ¿Qué voy a enseñar? ¿Cómo y con qué lo voy a hacer?” (Zubiría, 2010, p. 32).

Finalmente, es importante reconocer que todo contexto educativo plantea sus particularidades, en los cuales las contingencias y distintos personajes y personalidades proponen desafíos constantes. No todas las personas aprenden al mismo ritmo; los gustos y capacidades son diferentes, unos tienen problemas de comunicación, otros de aprendizaje, entre otros. Ante estas realidades, la pedagogía está llamada a entrar en acción, haciendo del acto educativo una oportunidad valiosa de aprendizaje y de desarrollo de habilidades y capacidades que materialicen las intencionalidades formativas de las instituciones, sin desestimar a la persona y sus particularidades.

\section{Bibliografía}

Berríos, F. (2012). Pedagogía en Teología: el aporte de Karl Rahner. Veritas, 26, pp, 187-196. https://doi.org/10.4067/S0718-92732012000100009

Castillo, M. y Gamboa, R. (2012) Desafíos de la educación en la sociedad actual. Revista Electrónica Diálogos Educativos, 24, (12), pp. 55-69.

Cencini, A. (2005) El árbol de la vida. Hacia una formación inicial y permanente. Madrid: Editorial San Pablo. 
Código de Derecho Canónico (1983). Corpus Iuris Canonici. http://www.vatican. va/archive/ESL0020/_INDEX.HTM

Conferencia Española de Religiosos (2015). Aspectos de la formación inicial. Madrid: Edita conferencia Española de religiosos.

Concilio Vaticano II. (1965). Documentos del Concilio Vaticano II. Decreto Perfectae Caritatis, sobre la renovación de la vida religiosa. Madrid: La Editorial Católica, S.A.

Congregación para los institutos de vida consagrada y las sociedades de vida apostólica (1990). Orientaciones sobre la formación en los institutos religiosos. Tomado de: http://www.vatican.va/roman_curia/congregations/ccscrlife/documents/ rc_con_ccscrlife_doc_02021990_directives-on-formation_sp.html

Creswell, J.W. (1998). Qualitative Inquiry and Research Desiamong Five Traditions. California: Thousand Oaks Sage.

Flórez, R. (2005). Pedagogía del conocimiento. Bogotá: Mc Graw Hill.

Giroux, H. (1990) Los profesores como intelectuales. Hacia una pedagogía critica del aprendizaje. Barcelona: Ediciones Paidós.

Grossman, P., Wilson, S. y Shulman, L. (2005). Profesores de sustancia: el conocimiento de la materia para enseñanza. Profesorado. Revista de Curriculum y Formación de Profesorado, 9 (2), pp. 1-25.

Hernández, S. (2010). Educación y ética. Revista Sociológica, 72, pp. 215-227.

Juan Pablo II, (1996). Vita Consecrata. Buenos Aires: Editorial Claretiana.

Ocaña, O. (2013). Modelos pedagógicos y teorías del aprendizaje. Bogotá: Ediciones de la U.

Mahecha, G., Cubillos, H., y Pico, A. (2018). Aportes de la dimensión espiritual al currículo de la ERE en el caso de la educación básica. Revista Electrónica Nuevas Búsquedas, 8, pp. 15-27.

Marín, J. (2016). La investigación en educación y pedagogía. Sus fundamentos epistemológicos y metodológicos. Bogotá: Universidad Santo Tomás.

Naranjo, S. y Moncada, C. (2019). Aportes de la Educación Religiosa escolar al cultivo de la espiritualidad humana. Revista Educación y Educadores, 22 (1), pp. 103-119. https://doi.org/10.5294/edu.2019.22.1.6

Páramo, P. (2013). La investigación en ciencias sociales: Estrategias de investigación. Bogotá D.C.: Universidad Piloto de Colombia. 
Pérez, J., González, Y., y Rodríguez, A. (2017). Aportes de la pedagogía crítica a la fundamentación metodológica de la teología de la liberación en América Latina. Revista Hojas y Hablas, 14, pp. 41-54. https://doi.org/10.29151/ hojasyhablas.n14a3

Pérez, J., Nieto-Bravo, J., y Santamaría-Rodríguez, J. (2019). La hermenéutica y la fenomenología en la investigación en ciencias humanas y sociales. Civilizar: Ciencias Sociales Y Humanas, 19(37).

Rodríguez, J. (2013). Una mirada a la pedagogía tradicional y humanista. Presencia universitaria, 3(5), pp. 36-45.

Santamaría, J., Quitián, E., y Orozco, A. (2016). Caracterización de una pedagogía de la teología en perspectiva crítico-liberadora. Reflexiones desde la pedagogía crítica y la teología de la liberación. Albertus Magnus, 7(2), pp. 213-237. https://doi.org/10.15332/s2011-9771.2016.0002.02

Vargas, J., Arcila, Y., y Robayo, A. (2017). La Teología de la Liberación y la Pedagogía del Oprimido, un camino hacia la emancipación. Guillermo de Ockham, 15(1), 10, pp. 103-107.

Zubiría, J. (2010). Los modelos pedagógicos. Hacia una pedagogía dialogante. Editorial pedagógica dialogante. Colombia. 